\title{
Características clínico-epidemiológicas da coinfecção por tuberculose e HIV e sua relação com o Índice de Desenvolvimento Humano no estado do Mato Grosso do Sul, Brasil
}

\section{Clinical and epidemiological characteristics of tuberculosis and HIV coinfection and the association with the Human Development Index in Mato Grosso do Sul State, Brazil}

Sueli Santiago Baldan', Antonio Sergio Ferraudo², Monica de Andrade ${ }^{3}$

' Universidade Federal de Mato Grosso do Sul, Coxim, Mato Grosso do Sul, Brasil

2 Universidade Estadual Paulista "Julio de Mesquita Filho", Jaboticabal, São Paulo, Brasil

3 Universidade de Franca, Franca, São Paulo, Brasil

\begin{abstract}
RESUMO
OBJETIVOS: Verificar os aspectos epidemiológicos da coinfecção por tuberculose (TB) e HIV, no estado do Mato Grosso do Sul, Brasil, e sua associação com o Índice de Desenvolvimento Humano (IDH). MATERIAIS E MÉTODOS: Estudo descritivo, do tipo ecológico, baseado em casos de coinfecção por TB e HIV, notificados no período de 2004 a 2013, e no IDH dos municípios no ano de 2010. Foram utilizados indicadores sociodemográficos e clínicos dos casos de coinfecção. Para testar a associação entre o percentual de coinfecção TB/HIV e o IDH dos municípios, foi realizada a análise logística de Poisson, e, para identificar a existência de autocorrelação espacial, foi calculado o índice de Moran. RESULTADOS: No período de estudo, foram notificados 10.179 casos de TB, dos quais 988 $(9,7 \%)$ apresentaram sorologia anti-HIV positiva. Em 2.985 (29,3\%) casos, o teste sorológico não foi realizado. Os casos de coinfecção TB/HIV ocorreram predominantemente em indivíduos do sexo masculino (68,9\%), com baixa escolaridade $(64,0 \%)$, na forma clínica pulmonar (69,5\%). A taxa de cura foi de $47,3 \%$, a de abandono de tratamento foi de 10,5\% e a de óbito por TB foi de 7,7\%. A análise espacial por meio do índice de Moran global mostrou a existência de autocorrelação espacial $(p<0,05)$, concordando com o resultado da análise de regressão de Poisson. CONCLUSÃO: Observou-se a associação entre casos de coinfecção por TB/HIV e IDH em áreas com maior densidade populacional, indicando a necessidade de adoção de estratégias específicas de acordo com as características de cada localidade.
\end{abstract}

Palavras-chave: Tuberculose; HIV; Epidemiologia; Desenvolvimento Humano.

\begin{abstract}
OBJECTIVES: To verify the epidemiological aspects of tuberculosis (TB) and HIV coinfection in Mato Grosso do Sul State, Brazil, and the association with the Human Development Index (HDI). MATERIALS AND METHODS: This is a descriptive ecological study based on cases of TB and HIV coinfection reported between 2004 and 2013, and the municipalities HDI in 2010. Sociodemographic and clinical indicators of the cases of TB/HIV coinfection were used. Poisson logistic analysis was conducted in order to test the association between the percentage of TB/HIV coinfection and the municipalities HDI; Moran's index was calculated to identify the existence of spatial autocorrelation. RESULTS: In the study period, 10,179 TB cases were reported, of which 988 (9.7\%) presented positive anti-HIV serology. For 2,985 (29.3\%) of notified cases, the serological test was not performed. TB/HIV coinfection cases predominantly occurred in males (68.9\%), with low educational level (64.0\%), and in the pulmonary clinical form (69.5\%). Cure rate was $47.3 \%$, treatment dropout rate was $10.5 \%$, and TB death rate was $7.7 \%$. Spatial analysis using global Moran's index showed the existence of spatial autocorrelation $(p<0.05)$, agreeing with the result of Poisson regression analysis. CONCLUSION: The association between cases of TB/HIV coinfection and HDI in areas with higher population density indicates the need to adopt specific strategies according to the characteristics of each locality.
\end{abstract}

Keywords: Tuberculosis; HIV; Epidemiology; Human Development.

\footnotetext{
Correspondência / Correspondence:

Sueli Santiago Baldan

Av. Marcio Lima Nantes, s/n. Bairro: Vila da Barra - CEP: 79400-000 - Coxim, Mato Grosso do Sul, Brasil - Tel.: +55 (67) 3291 -0202

E-mail:sueli.baldan@ufms.br
} 


\section{INTRODUÇÃO}

A tuberculose (TB) é uma doença infectocontagiosa tão antiga quanto a história da humanidade, causada pela bactéria Mycobacterium tuberculosis. Já a síndrome da imunodeficiência adquirida (aids), que data da década de 1980, é a doença causada pelo vírus HIV. A pandemia de aids causou grande impacto sobre o perfil clínico e epidemiológico da TB' . Atualmente, a infecção pelo HIV é o principal fator de risco para o desenvolvimento da $\mathrm{TB}$, e a doença é a principal causa de morte entre as pessoas vivendo com HIV/aids (PVHA $)^{2}$, pois em indivíduos com coinfecção $\mathrm{TB} / \mathrm{HIV}$, os riscos de falência de tratamento, de desenvolvimento de TB multidroga resistente (TBMDR) e de recidivas são maiores ${ }^{3,4}$.

Tanto a TB quanto a infecção pelo HIV são consideradas importantes problemas de saúde pública global, pois no ano de 2015, aproximadamente 10,4 milhões de pessoas adoeceram por TB, das quais 1,2 milhão eram PVHA. Ainda em 2015, 1,8 milhão de pessoas morreram devido à $T B$, incluindo 400.000 pessoas que eram HIV positivo ${ }^{5}$.

No Brasil, em 2015, foram notificados 81.137 casos de TB e, dentre esses, 73.221 eram casos novos (incidência 35,2 casos/100.000 habitantes), dos quais $15 \%$ eram HIV positivo. O país ocupa, atualmente, a $19^{a}$ posição em uma lista de 30 países com maior número de casos de coinfecção TB/HIV5 .

O estado de Mato Grosso do Sul apresentou, em 2015, uma taxa de incidência de TB de 28,4/100.000 habitantes, sendo que, em $71,1 \%$ dos casos, foi realizada a testagem para HIV, resultando em $9,6 \%$ de coinfecção TB/HIV ${ }^{6}$.

De acordo com o Ministério da Saúde, dentre as ações prioritárias para pessoas com TB é recomendado buscar o diagnóstico precoce de infecção pelo HIV. Caso se confirme a infecção, indica o tratamento antirretroviral. Para as PVHA, a recomendação refere-se ao diagnóstico precoce da TB, o tratamento adequado para $T B$, a identificação e o tratamento de infecção latente de TB (ILTB $)^{7}$. Esses cuidados são importantes, pois o tratamento da ILTB em portadores do HIV reduz significativamente $\mathrm{o}$ risco de desenvolvimento de $\mathrm{TB}^{8}$.

A End TB Strategy, estratégia global para prevenção, cuidado e controle da TB pós 2015, da Organização Mundial da Saúde (OMS), estabelece a redução do número de mortes por TB em $35 \%$ e a redução da incidência em 20\% até 2020, em relação aos indicadores de $2015^{8}$.

O impacto negativo da inter-relação entre a TB e $\circ$ HIV representa um desafio para $\bigcirc$ alcance das metas estabelecidas pela OMS, pois o diagnóstico da doença nos indivíduos HIV positivo costuma ser tardio, em decorrência de falhas de comunicação entre os serviços de referência para $O$ atendimento às PVHA e com TB. Outro fator que interfere negativamente na redução do número de mortes e de incidência de TB é o fato de que muitos indivíduos não se reconhecem em risco para o desenvolvimento de doenças, diminuindo a prática do autocuidado. Outros têm dificuldades de acesso aos serviços de saúde ou ainda adiam a procura de atendimento, para evitar receber um diagnóstico de aids?.

No período de 2000 a 2011 , houve uma importante redução na taxa de mortalidade por coinfecção TB/HIV no Brasil. No entanto, ocorreu um aumento na mortalidade por coinfecção TB/HIV nas Regiões Norte, Nordeste e Centro-Oeste. Esse fato reflete as diferenças socioeconômicas dessas regiões em relação às Regiões Sul e Sudeste, que apresentaram uma redução significativa na mortalidade².

A maioria dos casos de TB e de HIV ocorre em regiões com menores recursos econômicos, dificultando o diagnóstico, o tratamento e o controle da infecção. Além disso, apesar das grandes conquistas no tratamento tanto da TB como do HIV/aids, os serviços de saúde pública nem sempre conseguem atender às demandas para 0 controle das epidemias ${ }^{10}$.

Tendo em vista o fato de que a TB exerce um impacto negativo no crescimento econômico e no desenvolvimento social, torna-se necessário elaborar programas que permitam a redução das desigualdades sociais, favorecendo a melhoria dos indicadores da doença, interrompendo o ciclo que alimenta a pobreza e a exclusão social ${ }^{11}$.

Uma forma de investigar as desigualdades sociais e de saúde é por meio do Índice de Desenvolvimento Humano (IDH), composto por três indicadores: longevidade, educação e renda, que apontam as condições para uma sociedade ter "vidas longas e saudáveis, acesso a conhecimento, e de ter comando sobre os recursos de forma a garantir um padrão de vida digno"12. O Programa das Nações Unidas para o Desenvolvimento (PNUD) classifica o desenvolvimento humano, com base no IDH, em muito baixo $(0,000$ e $0,499)$, baixo $(0,500$ a 0,599$)$, médio $(0,600$ a 0,699$)$, alto $(0,700$ a 0,799$)$ e muito alto $(\geq 0,800)^{12}$.

Considerando a magnitude do problema que a coinfecção TB/HIV representa, não apenas no âmbito da saúde pública, mas também no social, o conhecimento dos diferentes aspectos relacionados à ocorrência de coinfecção TB/HIV favorece a elaboração de estratégias de controle nas diferentes esferas de gestão da saúde.

O objetivo deste estudo foi verificar os aspectos epidemiológicos da coinfecção TB/HIV, a associação entre casos de coinfecção TB/HIV e o IDH dos municípios e sua distribuição espacial no estado do Mato Grosso do Sul, Brasil.

\section{MATERIAIS E MÉTODOS}

Estudo descritivo, do tipo ecológico, de casos de coinfecção TB/HIV notificados em residentes no estado de Mato Grosso do Sul, no período de janeiro de 2004 a dezembro de 2013.

Mato Grosso do Sul localiza-se na Região CentroOeste brasileira, possui 79 municípios; porém o 
município de Paraíso das Águas, criado em 1 de janeiro de 2013, foi excluído do estudo por não constar como município naquele período. Portanto, fizeram parte da pesquisa 78 municípios.

Os dados de coinfecção TB/HIV foram obtidos do Sistema de Informação de Agravos de Notificação (Sinan) ${ }^{13}$, no mês de dezembro de 2016, e do Instituto Brasileiro de Geografia e Estatística (IBGE) ${ }^{14}$. As variáveis obtidas foram: sexo, faixa etária, raça, escolaridade, forma clínica, tipo de entrada, resultado da sorologia anti-HIV, condição de institucionalização, baciloscopia na primeira amostra e forma de encerramento do caso. $\bigcirc \mathrm{IDH}$ dos municípios para o ano de 2010 foi obtido com base na classificação adotada pelo PNUD ${ }^{12}$. Os dados demográficos foram extraídos do censo populacional realizado pelo IBGE em 2010.

Para testar a associação entre a coinfecção TB/HIV e o IDH dos municípios, foram calculados o coeficiente de incidência de TB (Cl-TB) e o percentual de coinfecção TB/HIV, no período. Calculou-se também a média das variáveis de interesse (Cl-TB; \% coinfecção TB/HIV e IDH dos municípios). A análise de correlação entre o percentual de coinfecção TB/HIV e o IDH dos municípios foi realizada por meio do teste de regressão de Poisson, utilizado o software STATISTICA, v7.0 da Statsoft.

Com o objetivo de verificar a existência de locais que apresentaram autocorrelação dos casos de coinfecção TB/HIV, foram calculados o índice de Moran e o indicador local de associação espacial (LISA). $\bigcirc$ teste de pseudossignificância foi calculado a partir de 99 permutações.

○ cálculo dos índices de Moran global e local, bem como o mapa temático mostrando essa distribuição, foram feitos no TerraView v4.2.2.

\section{RESULTADOS}

De um total de 10.179 casos de TB notificados no período, 988 (9,7\%) apresentaram sorologia anti-HIV positiva, sendo que, em 2.985 (29,3\%) casos notificados, o teste sorológico não foi realizado.

Quanto aos aspectos sociodemográficos dos indivíduos com sorologia positiva para HIV, houve predomínio do sexo masculino (68,9\%); 37,9\% dos indivíduos eram pardos e $37,2 \%$ brancos. A faixa etária com sorologia positiva para HIV predominante foi a de 20 a 39 anos $(51,2 \%)$ e com nível de escolaridade de até oito anos de estudo (64,0\%). Encontravam-se privados de liberdade $10,0 \%$ dos indivíduos (Tabela 1).

Quanto às características clínicas, $77,5 \%$ eram casos novos, 69,5\% apresentaram TB pulmonar e, 27,8\% tiveram baciloscopia de escarro positiva na primeira amostra. $O$ percentual de casos de TB curados foi de $47,3 \%$, sendo que $18,4 \%$ dos casos evoluíram para óbito por outras causas e 7,7\% foram a óbito por TB (Tabela 2).
Tabela 1 - Distribuição dos casos de coinfecção TB/HIV, de acordo com características sociodemográficas, no período de 2004 a 2013, Mato Grosso do Sul, Brasil

\begin{tabular}{lcc}
\hline \multicolumn{1}{r}{ Variável } & $N=988$ & $\%$ \\
\hline Sexo & 307 & 31,1 \\
Feminino & 681 & 68,9 \\
Masculino & & \\
Raça/cor & 50 & 5,1 \\
Amarela & 368 & 37,2 \\
Branca & 37 & 3,7 \\
Indígena & 374 & 37,9 \\
Parda & 91 & 9,2 \\
Preta & 68 & 6,9 \\
Ignorado & &
\end{tabular}

Faixa etária (anos)$$
\leq 9
$$

10 a 19

20 a 39

40 a 59

$\geq 60$

Escolaridade (em anos de estudo)

0 a 4

5 a 8

9 a 11

$\geq 12$

Ignorado

Institucionalização

Não institucionalizado

Asilo

Hospital psiquiátrico

Orfanato

Presídio

Outro

lgnorado

250

25,3 (DATASUS), 2016. 
Tabela 2 - Características clínicas dos casos de coinfecção TB/HIV, no período de 2004 a 2013, Mato Grosso do Sul, Brasil

\begin{tabular}{lcc}
\multicolumn{1}{l}{ Variável } & $\mathrm{N}=988$ & $\%$ \\
\hline Forma & & \\
Extrapulmonar & 234 & 23,7 \\
Pulmonar & 687 & 69,5 \\
Pulmonar + extrapulmonar & 67 & 6,8
\end{tabular}

Tipo de entrada

Caso novo

766

77,5

Recidiva

92

Reingresso após abandono

84

Transferência

38

Não sabe

8

Primeira baciloscopia de escarro

Positivo

275

Negativo

Não realizado

Encerramento

$\begin{array}{lcc}\text { Abandono } & 104 & 10,5 \\ \text { Cura } & 467 & 47,3 \\ \text { Óbito por TB } & 76 & 7,7 \\ \text { Óbito por outras causas } & 182 & 18,4 \\ \text { TB multirresistente } & 3 & 0,3 \\ \text { Transferência } & 75 & 7,6 \\ \text { Ignorado } & 81 & 8,2\end{array}$

Fonte: DATASUS, 2016.

A regressão logística de Poisson mostrou associação entre os casos de coinfecção TB/HIV e o IDH $(p<0,05)$.

Quanto à distribuição das taxas de coinfecção TB/HIV nos municípios de Mato Grosso do Sul, foi construído um mapa temático (Figura 1), onde é possível identificar uma distribuição heterogênea dos casos de coinfecção TB/HIV.

$\bigcirc$ índice de Moran global mostrou a existência de autocorrelação espacial $(p<0,05)$. $O$ índice de Moran local foi utilizado para detalhar os municípios onde essa dependência pode estar ocorrendo. $\mathrm{Na}$ figura 2, encontram-se destacados os locais contendo autocorrelação espacial local $(p<0,05)$.

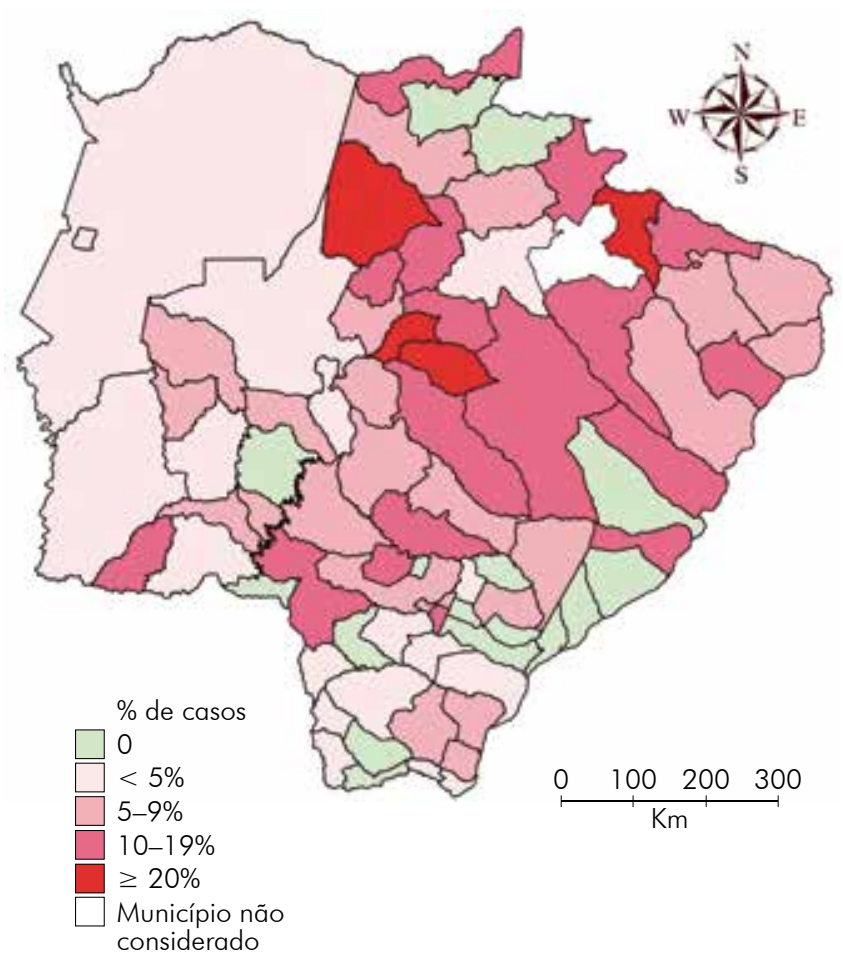

Figura 1 - Mapa temático de casos de coinfecção TB/HIV, Mato Grosso do Sul, Brasil, 2004-2013

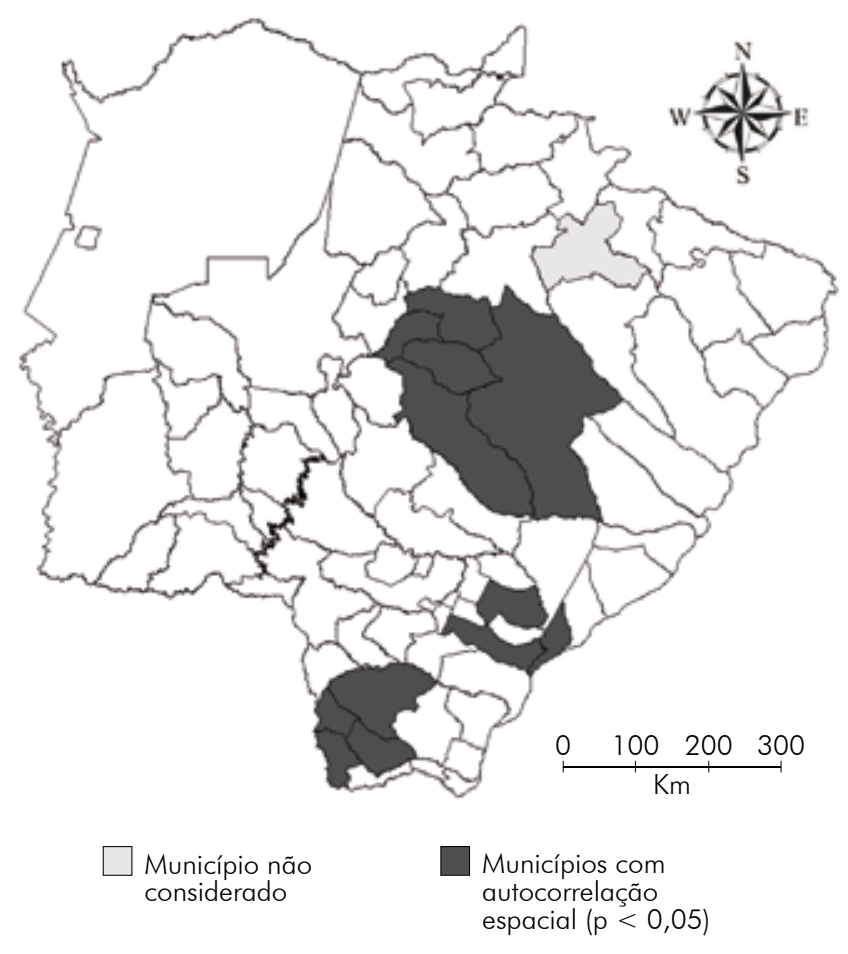

Figura 2 - Distribuição espacial dos agrupamentos de casos de coinfecção TB/HIV, Mato Grosso do Sul, Brasil, 2004-2013

Para facilitar a visualização da distribuição dos municípios, por meio do IDH e dos casos de coinfecção, com base no percentual de coinfecção TB/HIV, foi elaborado um mapa temático proporcional (Figura 3). 


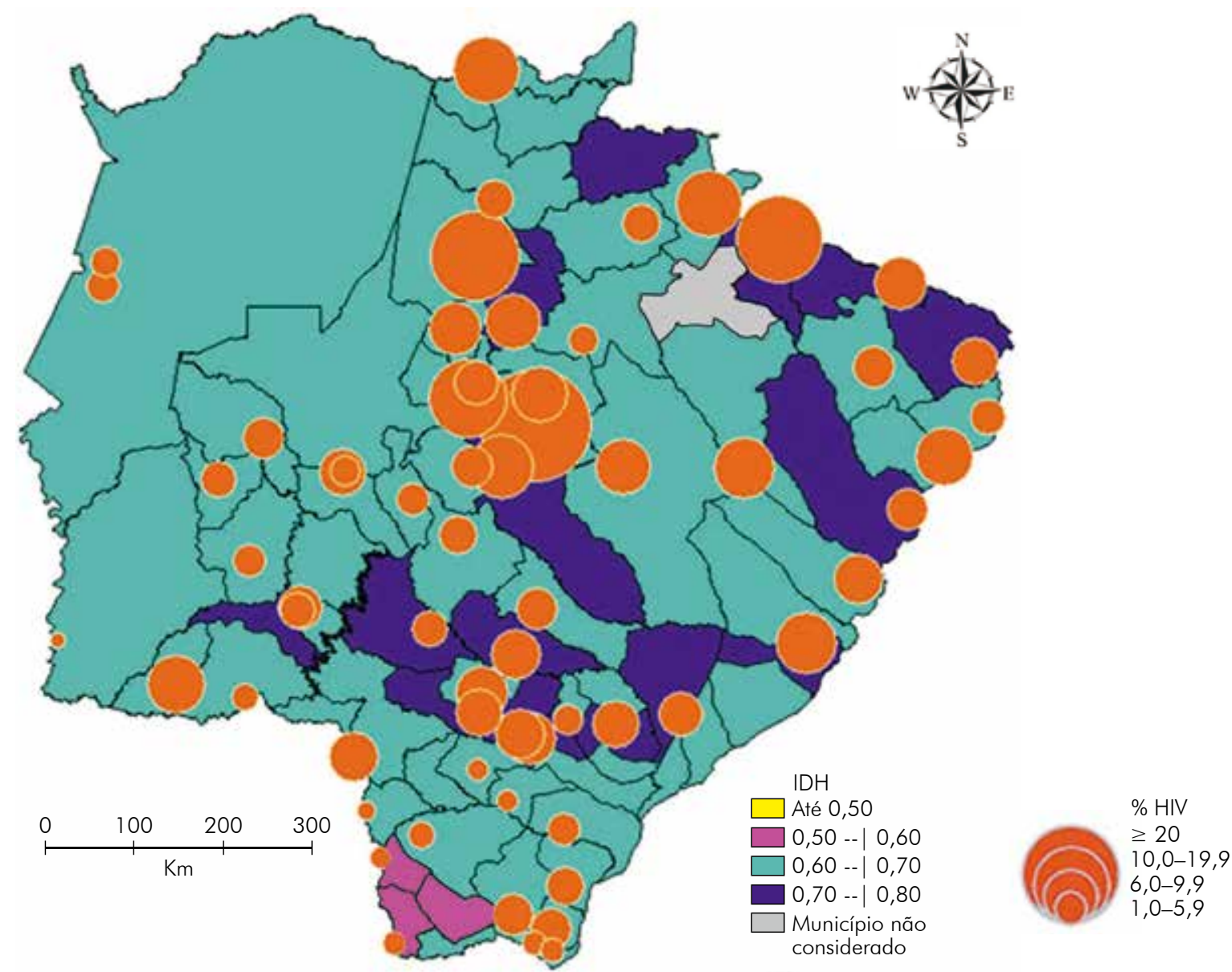

Figura 3 - Mapa proporcional da distribuição dos municípios, IDH e coinfecção TB/HIV, Mato Grosso do Sul, Brasil, 2004-2013

\section{DISCUSSÃO}

A OMS começou a monitorar a integração de serviços de TB/HIV em 2004, como forma de diminuir o impacto do HIV entre indivíduos com TB, recomendando, entre outras estratégias, a realização do teste de HIV de rotina a todos os pacientes com $T B$, àqueles com sinais e sintoma de TB e, também, aos parceiros de pacientes com TB/HIV positivos. Essa medida permitiu salvar, aproximadamente, 5,8 milhões de vidas em todo o mundo 5 .

Neste estudo, a porcentagem de pessoas diagnosticadas com a coinfecção TB/HIV (9,7\%), no Mato Grosso do Sul, é a mesma encontrada no Brasil ${ }^{6}$ em 2015. A porcentagem de coinfecção TB/HIV nesse Estado é superior a alguns países da Europa, como Espanha (6,2\%), Inglaterra (7,4\%), Áustria $(1,7 \%)$, França $(5,6 \%)$ e Bélgica $(5,6 \%)$; no entanto é inferior a Portugal $(14,6 \%)^{15}$.

Quando comparada a outros estados brasileiros, a porcentagem no Mato Grosso do Sul é maior que em Minas Gerais $(8,3 \%)^{16}$ e menor que em Pernambuco $(10,5 \%)^{10}$. O município de Porto Alegre, estado do Rio Grande do Sul, apresentou uma porcentagem de coinfecção TB/HIV de 23,6\% no período de 2007 a $2011^{17}$. As variações nesses resultados podem ocorrer devido a diferenças no cálculo adotado em cada estudo, assim como por variação real no número de casos de coinfecção TB/HIV.

É preciso considerar a existência de fatores associados à subnotificação de TB e aids ${ }^{18}$, como o fato de que, muitas vezes, o profissional que realiza a notificação desconhece as doenças a notificar ou ocorrem problemas no fluxo da notificação. Esses problemas podem ocorrer por mudanças na definição de caso e falta de comunicação entre os profissionais, que não notificam por acreditar que outro profissional já realizou a notificação.

Em um estudo sobre subnotificação de casos de TB a partir da vigilância do óbito, os resultados indicaram uma relação entre subnotificação e indivíduos do sexo feminino, pessoas com menos de 60 anos de idade e com escolaridade inferior a oito anos de estudo ${ }^{19}$.

No período de realização deste estudo, houve um percentual de casos de TB diagnosticados, nos quais não se realizou o teste anti-HIV $(29,3 \%)$, que é considerado alto. Esse resultado pode indicar a necessidade de maiores esforços de profissionais da área da saúde e gestores, para que todos tenham acesso ao diagnóstico e tratamento adequados.

Houve um predomínio de casos em indivíduos do sexo masculino (68,9\%). Esse resultado é 
corroborado por estudos realizados na Região Nordeste brasileira9, no estado de Minas Gerais ${ }^{16}$ e na União Europeia $^{15}$.

Os fatores que interferem na maior ocorrência de casos em homens podem estar relacionados a diversas condições, seja de ordem biológica, cultural e organizacional, devido à inflexibilidade nos horários de atendimento, o que dificulta o acesso dos trabalhadores, ou ainda a possibilidade de subdiagnóstico em mulheres 20,21 .

percentual identificado de pessoas com a coinfecção TB/HIV, no presente estudo, foi semelhante entre pardos e brancos. De acordo com dados do IBGE$^{22}$, a população do Mato Grosso do Sul é composta por $47,3 \%$ de pessoas brancas, $43,6 \%$ pardas, 4,9\% pretas, 3,0\% indígenas e 1,2\% amarelas. Em um estudo realizado em um hospital de referência de Campo Grande, capital de Mato Grosso do Sul, houve predominância de coinfecção TB/HIV em indivíduos brancos ${ }^{23}$.

Este estudo demonstrou que há um maior número de casos de coinfecção TB/HIV em indivíduos na faixa etária de 20 a 39 anos, refletindo uma maior proporção de TB nesse grupo ou realização de testes de HIV mais frequente no referido grupo. ○ estilo de vida da população nessa faixa etária, com comportamentos vulneráveis, pode resultar em maior exposição ao HIV e ao M. tuberculosis ${ }^{24}$. Estudos realizados no estado de São Paulo25, na Região Nordeste brasileira ${ }^{10}$, em Porto Alegre ${ }^{17}$ e no Brasil26, corroboram os resultados obtidos neste estudo.

nível educacional representa um determinante social da saúde, uma vez que o acesso à educação influencia na capacidade do sujeito assimilar as informações de promoção de saúde, de prevenção de doenças e na adesão ao tratamento. Na presente pesquisa, observou-se uma baixa escolaridade da população estudada. Tal fato também foi identificado em um estudo realizado no estado do Maranhão, - qual propôs que a baixa escolaridade pode levar a condições desfavoráveis de vida e emprego, contribuindo para um ambiente propício ao incremento da prevalência de $\mathrm{TB}^{24}$.

Para pessoas que vivem em instituições como asilos, orfanatos ou presídios, a TB é um sério problema de saúde, tendo em vista as condições ambientais desfavoráveis em que estão inseridas. $\bigcirc$ percentual de casos de coinfecção TB/HIV em pessoas privadas de liberdade foi de 10,0\%. Esse valor é superior ao observado na Região Nordeste ${ }^{10}$. Em um estudo realizado no Mato Grosso do Sul, para verificar a infecção latente por TB entre pessoas privadas de liberdade, constatou-se uma baixa incidência da doença nessa população, quando comparada a outros estados e países ${ }^{27}$

Quanto às características clínicas, o maior número de registros de casos novos de TB e de TB pulmonar, observados neste estudo, está corroborado por diversas pesquisas realizadas no Brasil'1,24,25,26. $O$ percentual de casos de TB extrapulmonar aqui identificados diverge de outros estudos. Embora a TB extrapulmonar não seja considerada como um fator de risco em relação à transmissão da doença, tem ocorrido um aumento da sua incidência, fato esse ligado à epidemia do HIV. Os estados do Ceará $^{\text {e Maranhão }}{ }^{24}$ apresentaram um maior número de casos de TB extrapulmonar em indivíduos coinfectados.

Embora exista no mercado exames mais precisos para diagnóstico da TB, a baciloscopia ainda é um importante recurso para o diagnóstico da doença, devido ao seu baixo custo e simplicidade. Nesta pesquisa, notou-se um elevado número de casos em que não foi realizado o exame baciloscópico. Um estudo feito na Região Nordeste do País também apresentou um número elevado de casos em que a baciloscopia não foi realizada ${ }^{10}$. Considerando que os exames de raio $X$ e histológicos têm baixa especificidade para $O$ diagnóstico de TB, faz-se necessário investigar os motivos para uma elevada proporção de casos sem confirmação baciloscópica ${ }^{5}$.

O Ministério da Saúde preconiza uma taxa de abandono inferior a $5,0 \%$ e uma taxa de cura de $85,0 \%$ para a $\mathrm{TB}^{7}$. Este estudo apontou para uma taxa de cura entre os casos de TB/HIV abaixo do recomendado, assim como taxas de abandono de tratamento e de mortalidade por TB/HIV elevadas. Resultados similares foram encontrados no Ceará ${ }^{\mathrm{e}}$ no município de São Paulo20. $O$ número elevado de mortes por TB é um indicador de um sistema de saúde com problemas de eficiência, pois a TB é uma doença tratável, se houver diagnóstico precoce e tratamento adequado.

A informação sobre a forma de encerramento do tratamento constou como ignorada em $8,2 \%$ dos casos. Isso sugere a necessidade de aprimorar o sistema de preenchimento da ficha de investigação e de digitação dos dados, para evitar erros no diagnóstico da situação de saúde.

Embora a ocorrência de TB esteja estreitamente relacionada à pobreza, resultados encontrados no presente estudo mostraram que os municípios com maior IDH apresentaram uma taxa maior da coinfecção TB/HIV. A constatação de que o menor número de casos coincide com as áreas de baixos valores do IDH também foi observada no estado de São Paulo ${ }^{25}$ e no município do Rio de Janeiro ${ }^{28}$. A existência de bolsões de pobreza e de áreas endêmicas pode ser uma explicação para esse fenômeno ${ }^{17}$.

Outro fator a ser considerado é a possibilidade de subnotificações de casos nos municípios onde houve menor número de casos identificados, ou ainda uma menor oferta do teste sorológico para o HIV. 
uso de instrumentos de análise espacial favorece a identificação de áreas com maior risco; assim como a observação da correlação dessas áreas com indicadores sociais pode subsidiar estratégias e intervenções específicas e, também, permite priorizar recursos que contribuam para redução de tais diferenças entre áreas ${ }^{29}$.

Mato Grosso do Sul possui quatro macrorregiões de saúde: Campo Grande (34 municípios e 1.364.668 habitantes), Corumbá (dois municípios e 125.179 habitantes), Dourados (33 municípios e 758.680 habitantes) e Três Lagoas $(10$ municípios e 256.561 habitantes) ${ }^{30}$. Por meio da análise espacial, pôde-se observar um agrupamento de municípios com maior ocorrência de casos de coinfecção TB/HIV na Macrorregião de Campo Grande, área que apresenta maior densidade demográfica. Esse resultado coincide com o encontrado em um estudo realizado na Região Nordeste do Brasil ${ }^{10}$, indicando a necessidade de avaliar a oferta de bens e serviços, a circulação de pessoas, de maneira a garantir um melhor acesso aos serviços de saúde.

As iniquidades socioeconômicas relacionadas a movimentos migratórios, a rápida urbanização e o aumento populacional geram desigualdades sociais, como a dificuldade de acesso a alimentos de qualidade, desnutrição, moradias inadequadas, pobreza e barreiras culturais ou geográficas e de acesso aos serviços de saúde ${ }^{31}$, favorecendo o surgimento de agravos à saúde, como a TB e o HIV.

As intervenções biomédicas contribuem para o controle da TB; no entanto a implantação de medidas com abordagem biológica, social, econômica e ambiental é imprescindível para a obtenção de resultados satisfatórios ${ }^{32}$.

Há que se remover as barreiras sociais, financeiras e dos sistemas de saúde que impedem o diagnóstico precoce do HIV, a oferta de terapia antirretroviral a todas as pessoas com HIV, a triagem de todos os indivíduos HIV-positivos para a TB e a realização de terapia preventiva com isoniazida, quando há uma forte suspeita de infecção latente ${ }^{33}$.

Compreender os diferentes fatores que envolvem a coinfecção TB/HIV favorece a adoção de medidas eficazes para o controle da doença. Para isso, são necessários estudos retrospectivos sobre padrões de distribuição espacial e temporal de doenças, utilizando-se dados secundários. $\bigcirc$ uso de dados secundários pode contribuir para o diagnóstico de saúde de uma população, no entanto possui limitações relacionadas à subnotificação de casos, ao preenchimento incorreto de fichas de notificação, demora na atualização do sistema de informação, entre outras.
É importante considerar, ainda, que estudos ecológicos estão sujeitos à ocorrência de um viés de agregação, fenômeno em que "a observação da existência de uma relação entre duas variáveis no nível agregado não implica, necessariamente, que essa relação se mantenha no nível individual"34.

trabalho integrado entre os serviços de atendimento à TB e ao HIV, assim como a capacitação dos responsáveis pelo preenchimento das fichas de notificação e alimentação do Sinan são fundamentais para um melhor acompanhamento da situação de saúde da comunidade.

\section{CONCLUSÃO}

Os resultados obtidos indicaram que, no período investigado, 9,7\% dos indivíduos com TB apresentavam coinfecção com o HIV; porém, em 29,3\% dos casos notificados, a investigação da situação sorológica não foi realizada.

percentual de cura dos casos de TB no período de estudo foi inferior ao preconizado pelo Ministério da Saúde, a taxa de abandono do tratamento identificada ficou acima do esperado e o percentual de óbitos em decorrência da TB mostrou-se elevado, considerando-se que pode ser evitado.

A associação entre casos de coinfecção TB/HIV e $\mathrm{IDH}$, em áreas com maior densidade populacional, indicou a necessidade de adoção de estratégias específicas de acordo com as características das localidades.

Os resultados do presente estudo podem contribuir para a elaboração de estratégias de controle, com vistas a melhorar os serviços de saúde, favorecendo a redução da morbimortalidade por $\mathrm{TB} / \mathrm{HIV}$, assegurando maior qualidade de vida à população.

A realização de novos estudos, em parceria com os serviços de atenção à saúde e outros setores da sociedade, como educação, infraestrutura, meio ambiente e desenvolvimento social, podem contribuir para a melhor compreensão do impacto do desenvolvimento humano na coinfecção TB/HIV.

\section{AGRADECIMENTOS}

Aos professores Dr. José de Paula Silva, da Universidade Estadual de Minas Gerais, e Dr. Salvador Bocaletti Ramos, da Universidade de Franca, pelas sugestões para melhoria na análise e apresentação dos dados.

\section{CONFLITOS DE INTERESSE}

Os autores declaram que não há conflitos de interesse. 


\section{REFERÊNCIAS}

1 Pires Neto RJ, Gadelha RRM, Herzer TL, Peres DA, Leitão TMJS, Façanha MC, et al. Características clínico-epidemiológicas de pacientes com coinfecção HIV/tuberculose acompanhados nos serviços de referência para HIV/AIDS em Fortaleza, Ceará, entre 2004 e 2008. Cad Saude Coletiva. 2012 abr;20(2):244-9.

2 Lima MS, Martins-Melo FR, Heukelbach J, Alencar $\mathrm{CH}$, Boigny RN, Ramos Júnior AN. Mortality related to tuberculosis-HIV/AIDS co-infection in Brazil, 2000-2011: epidemiological patterns and time trends. Cad Saude Publica. 2016 Oct;32(10): e00026715.

3 Lo HY, Suo J, Chang HJ, Yang SL, Chou P. Risk factors associated with death in a 12-month cohort analysis of tuberculosis patients. Asia Pac J Public Health. 2015 Mar;27(2):NP758-68.

4 Barbosa EL, Levino A. Análise da coinfecção TB/ HIV como fator de desenvolvimento da tuberculose multidroga resistente: uma revisão sistemática. Rev Pan-Amaz Saude. 2013 dez;4(4):57-66.

5 World Health Organization. Global tuberculosis report 2016. Geneva: WHO; 2016.

6 Ministério da Saúde (BR). Secretaria de Vigilância em Saúde. Perspectivas brasileiras para o fim da tuberculose como problema de saúde pública. Bol Epidemiol. 2016;47(13):1-15.

7 Ministério da Saúde (BR). Secretaria de Vigilância em Saúde. Departamento de Vigilância das Doenças Transmissíveis. Panorama da tuberculose no Brasil: indicadores epidemiológicos e operacionais. Brasília: Ministério da Saúde; 2014.

8 World Health Organization. The end TB strategy. Global strategy and targets for tuberculosis prevention, care and control after 2015. Document WHO/HTM/TB 2015.19. Geneva: WHO; 2015.

9 Guimarães RM, Lobo AP, Siqueira EA, Borges TFF, Melo SCC. Tuberculose, HIV e pobreza: tendência temporal no Brasil, Américas e mundo. J Bras Pneumol. 2012 jul-ago;38(4):511-7.

10 Barbosa IF, Costa ICC. Estudo epidemiológico da coinfecção tuberculose-HIV no Nordeste do Brasil. Rev Patol Trop. 2014 jan-mar;43(1):27-38.

11 Marques M, Ruffino-Netto A, Marques AMC, Andrade SMO, Silva Baldomero AK, Pontes ERJC. Magnitude da tuberculose pulmonar na população fronteiriça de Mato Grosso do Sul (Brasil), Paraguai e Bolívia. Cad Saude Publica. 2014 dez;30(12):2631-42.

12 Programa das Nações Unidas para 0 Desenvolvimento. Índice de desenvolvimento humano municipal Brasileiro. Brasília: PNUD/IPEA/ FJP; 2013. (Atlas do desenvolvimento humano no Brasil, 2013).
13 Ministério da Saúde (BR). Sistema de Informação de Agravos de Notificação - SINAN [Internet]. Brasil: Ministério da Saúde; 2016 [citado 2016 dez 16]. Disponível em: http://dtr2004.saude.gov.br/ sinanweb/tabnet/dh? sinannet/tuberculose/bases/ tubercbrnet.def.

14 Instituto Brasileiro de Geografia e Estatística. Sinopse do censo demográfico 2010: Brasil. Rio de Janeiro: IBGE; 2011.

15 Pimpin LN, Drumright ME, Kruijshaar I, Abubakar $B$, Rice V, Delpech V, et al. Tuberculosis and HIV co-infection in European Union and European Economic Area countries. Eur Respir J. 2011 Dec;38(6): 1382-92

16 Augusto CJ, Carvalho WS, Gonçalves AD, Ceccato MGB, Miranda SS. Características da tuberculose no estado de Minas Gerais entre 2002 e 2009. J Bras Pneumol. 2013 mai-jun;39(3):357-64.

17 Peruhype RC, Acosta LMW, Ruffino-Netto A, Oliveira MMC, Palha PF. Distribuição da tuberculose em Porto Alegre: análise da magnitude e coinfecção tuberculose-HIV. Rev Esc Enferm USP. 2014 dez;48(6): 1035-43.

18 Santos ML. Fatores associados à subnotificação de TB e aids, durante os anos de 2001 a 2010, a partir do Sinan [dissertação]. Recife (PE): Centro de Pesquisas Aggeu Magalhães, Fundação Oswaldo Cruz; 2014. 62 p.

19 Romero ROG, Ribeiro CMC, Sá LD, Villa TCS, Nogueira JA. Subnotificação de casos de tuberculose a partir da vigilância do óbito. Rev Eletr Enferm. 2016 jun;18:e1161.

20 Hino P, Takahashi RF, Bertolozzi MR, Egry EY. Coinfecção de Tb/HIV em um distrito administrativo do Município de São Paulo. Acta Paul Enferm. $2012 ; 25(5): 755-61$.

21 Assis MMA, Jesus WLA. Acesso aos serviços de saúde: abordagens, conceitos, políticas e modelo de análise. Cienc Saude Coletiva. 2012 nov; $17(11): 2865-75$.

22 Instituto Brasileiro de Geografia e Estatística. População residente, por cor ou raça, segundo a situação do domićlio, o sexo e a idade [Internet]. Rio de Janeiro: IBGE; 2015 [citado 2015 out 24]. Disponível em: http://www.sidra.ibge.gov.br/bda/ tabela/protabl.asp? $c=3175 \& z=c d \& o=7 \& i=p$.

23 Cheade MFM, Ivo ML, Siqueira PHGS, Sá RG, Honer MR. Caracterização da tuberculose em portadores de HIV/AIDS em um serviço de referência de Mato Grosso do Sul. Rev Soc Bras Med Trop. 2009 marabr;42(2): 1 19-25.

24 Santos Neto M, Silva FL, Sousa KR, Yamamura $M$, Popolin MP, Arcêncio RA. Perfil clínico e epidemiológico e prevalência da coinfecção tuberculose/HIV em uma regional de saúde no Maranhão. J Bras Pneumol. 2012 novdez;38(6):724-32. 
25 Pimenta ATM, Rodrigues Júnior AL, Ruffino Netto A. Geoepidemiologia da comorbidade AIDS/ Tuberculose no estado de São Paulo - Brasil - 1996 a 2005. Rev Caminhos Geogr. 2012 mar;13(41):68-79.

26 Rodrigues-Júnior AL, Ruffino-Netto A, Castilho EA. Distribuição espacial do Índice de Desenvolvimento Humano, da infecção pelo HIV e da comorbidade aids-tuberculose: Brasil, 1982-2007. Rev Bras Epidemiol. 2014;17 supl 2:204-15.

27 Carbone ASC, Paião DSG, Sgarbi RVE, Lemos EF, Cazanti RF, Ota MM, et al. Active and latent tuberculosis in Brazilian correctional facilities: a cross-sectional study. BMC Infect Dis. 2015 Jan; 15:24.

28 Pereira AGL, Medronho RA, Escosteguy CC, Valencia LIO, Magalhães MAFM. Distribuição espacial e contexto socioeconômico da tuberculose, Rio de Janeiro, Brasil. Rev Saude Publica. 2015 ago;49:48.

29 Oren E, Narita M, Nolan C, Mayer J. Neighborhood socioeconomic position and tuberculosis transmission: a retrospective cohort study. BMC Infect Dis. 2014 Apr; 14:227.
30 Mato Grosso do Sul. Secretaria Estadual de Saúde. Resolução n. 90/SES/MS, de 10 de dezembro de 2013. Definição do Regimento Interno para estabelecer as competências, organização e funcionamento da Comissão Estadual de Farmacoterapia - COMEFA. Diário Oficial, Campo Grande (MS), 2014 nov 11; n. 8.798. p. 10.

31 Hargreaves JR, Boccia D, Evans CA, Adato $M$, Petticrew M, Porter JDH. The social determinants of tuberculosis: from evidence to action. Am J Public Health. 2011 Apr;101(4):654-62.

32 Ortblad KF, Salomon JA, Bärnighausen T, Atun R. Stopping tuberculosis: a biosocial model for sustainable development. Lancet. 2015 Dec;386(10010):2354-62.

33 Godfrey-Faussett P, Ayles $H$. Why are people living with HIV still dying of tuberculosis? Lancet. 2016 Mar;387(10024):1141-3.

34 Silva JFS, Silva AMC, Lima-Luz L, Aydos RD, Mattos IE. Correlação entre produção agrícola, variáveis clínicas-demográficas e câncer de próstata: um estudo ecológico. Cienc Saude Coletiva. 2015 sep;20(9):2805-12.

Recebido em / Received: 12/10/2016 Aceito em / Accepted: 6/3/2017 